\title{
Theory and Numerical Simulation of Deep Rock Mass Based on a Non-Euclidean Model
}

\author{
Yingji Bao $\mathbb{i}^{1,2}$ and Binsong Jiang $\mathbb{D}^{1}$ \\ ${ }^{1}$ State Key Laboratory for Geomechanics and Deep Underground Engineering, China University of Mining and Technology, \\ Xuzhou 221116, China \\ ${ }^{2}$ School of Transportation Engineering, Jiangsu Vocational Institute of Architectural Technology, Xuzhou 221116, China \\ Correspondence should be addressed to Binsong Jiang; 10388@jsjzi.edu.cn
}

Received 27 September 2021; Revised 10 November 2021; Accepted 18 January 2022; Published 14 February 2022

Academic Editor: Tongguang Ni

Copyright (C) 2022 Yingji Bao and Binsong Jiang. This is an open access article distributed under the Creative Commons Attribution License, which permits unrestricted use, distribution, and reproduction in any medium, provided the original work is properly cited.

\begin{abstract}
Rock masses existing in nature may experience closed internal stress under the action of geological structures. This closed internal stress makes the deformation of the rock mass incompatible, so classic continuum theory is not suitable for analyzing the stress and deformation of rock masses. In this study, a non-Euclidean model for rock masses was established based on differential geometry. By choosing the non-Euclidean parameter as the internal variable, a thermodynamic model was constructed. Then, numerical computation based on the non-Euclidean model was applied to a circular tunnel in a deep rock mass. The distribution of internal stress and the effects of rock parameters on the distribution of internal stress were analyzed. Through our research, we conclude that the stress field of the deep rock mass consists of classical stress and internal stress and that the internal stress shows a distinct wavy behavior. The radius of the fractured zone decreases with an increasing Young's modulus $E$ and Poisson's ratio $v$, but increases with increasing non-Euclidean parameter $\xi$.
\end{abstract}

\section{Introduction}

In classical continuum theories of rock mechanics, a rock is a continuous, simply connected body that undergoes deformation, and the deformation satisfies the equations of compatibility. The metric tensor for measuring the distances between material particles in the reference configuration is Euclidean. However, rock masses existing in nature may contain closed internal stress. The internal stress makes the deformation of the rock incompatible. Therefore, the classical continuum theory is not suitable for analyzing the incompatible deformation of rock masses [1-5]. It is necessary to introduce non-Euclidean space to describe the incompatible deformation, and differential geometry will be used to describe the closed internal stress.

The relationship between the continuum theory and mathematical theory of differential geometry has been studied by many researchers. Kondo [6] first recognized the relationship between dislocation theory and non-
Riemannian theory. Later, Anthony [7] pointed out the relationship of differential geometry to disclinations. Then, Kroner [8] completed the underlying theory for defects and differential geometry, including dislocations and extra matter. These investigations established a relation between the parameters of non-Euclidean geometry and elastic strain, bend twist, and quasiplastic strain of defect theory [9]. The first non-Euclidean continuum model to describe the stress-field distribution around underground working was developed by Myanikov and Guzev [10]. A modification of this model was presented by Guzev and used to describe different rock phenomena, such as zonal disintegration and anomalous deformation of rock samples [11-15]. Investigations were also undertaken by other researchers, and a non-Euclidean continuum model was proposed to investigate the zonal disintegration phenomenon of surrounding rocks by Zhou and Qian [16-19]. These existing models assume that the undeformed body is in three-dimensional 
Euclidean space. However, there is closed internal stress in the rock mass.

Suppose there is a flat plate in two dimensions. Due to uneven heating and mutual expansion, thermal stress will be generated, and the uneven temperature field causes internal stress in the plate. If we cut the whole plate into tiny elements and release each tiny element into its stress-free natural state, then the flat plate composed of various elements can no longer be in two-dimensional Euclidean space. However, if the flat plate is allowed to warp, it will bulge and become a curved surface. The plate is a two-dimensional Riemannian space immersed in three-dimensional Euclidean space $R^{3}$. According to this scenario, the undeformed rock mass with closed internal stress should be regarded as a manifold.

In this study, it is assumed that the undeformed rock mass with closed internal stress was a manifold. A nonEuclidean model of rock masses was established based on differential geometry. By choosing the non-Euclidean parameter as the internal variable, a thermodynamic model was constructed. Then, numerical computation based on the non-Euclidean model was applied to a circular tunnel in a deep rock mass. The distribution of internal stress and the effects of rock parameters on the distribution of internal stress were then analyzed.

\section{Differential Geometry Method}

The initial state of the rock is as a manifold. Moreover, the manifold is an imagined state of undeformed rock that occupies a compact and simply connected region. Consider a material point $p$ in the undeformed body and an $\mathrm{m}$-dimensional manifold $M$ that is referred to as a configuration. We choose the configuration $\mathrm{p} \in \mathrm{U} \subset \mathrm{M}$ at time $t=t 0$ as a reference configuration, such that the subsequent deformation and motion of the current configuration are given by $q \in V \subset N$ at time $t=t$ with respect to the reference configuration [14] (Figure 1).

For any $p \in M$, there exists a neighborhood $U$ of $p$, such that $U$ is homeomorphic to an open set in $R^{m}$, i.e., the homeomorphism is $\varphi_{U}: \mathrm{U} \longrightarrow \varphi_{U}(\mathrm{U})$.

$$
X^{i}=\varphi_{U}(p)^{i},
$$

where $X^{i}$ is the local coordinate of the point $p \in U$.

For any $q \in N$, there exists a neighborhood $V$ of $q$, such that $V$ is homeomorphic to an open set in $R^{n}$, i.e., the homeomorphism is $\psi_{V}: v \longrightarrow \psi_{V}(\mathrm{~V})$.

$$
x^{i}=\psi_{V}(q)^{i}
$$

where $x^{i}$ is the local coordinate of the point $q \in V$.

The deformation of the body is a continuous map from the initial configuration smooth manifold $M$ to the final configuration smooth manifold $N . F: M \longrightarrow N$. Compatible coordinate charts $\left(U, \varphi_{U}\right)$ exist at points $p \in M$ and $\left(V, \psi_{V}\right)$ at $f(p) \in N$, such that the map

$$
\psi_{V} \circ F \circ \varphi_{U}^{-1}: \varphi_{U}(U) \longrightarrow \psi_{V}(V),
$$

is $C^{\infty}$ at the point $\varphi_{U}(p)$.

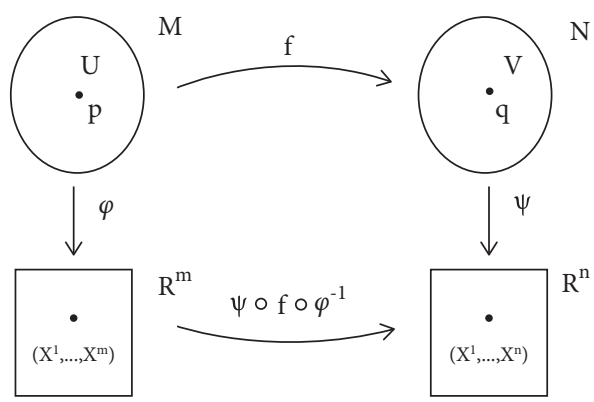

FIgURE 1: The initial configuration and final configuration. $M$ is the manifold of the undeformed body. $N$ is the manifold of the deformed body.

Moreover, smooth maps $F$ between $M$ and $N$ induce linear maps between tangent spaces and between cotangent spaces. $F: M \longrightarrow N, p \in M$, and $q=F(p) \in V$. $F_{*}$ is a linear map of the tangent space induced by $F$. We define the map $F_{*}$ : $T_{p} M \longrightarrow T_{q} N$ as follows (Figure 2):

$$
F_{*}(v)(f)=v\left(f^{\circ} F\right), \quad \forall f \in C_{F(p)}^{\infty} .
$$

The adjoint map of $F_{*}$ is $F^{*}: T_{p}^{*} M \longrightarrow T_{q}^{*} N$ which is called the differential map induced by $F$.

$$
F^{*}(d f)=d(f \circ F), \quad d f \in T_{q}^{*} N .
$$

$X^{i}$ and $x^{i}$ are the local coordinates of points $p$ and $q$. The deformation map $F$ can be expressed near $p$ by the functions

$$
x^{\alpha}=F^{\alpha}\left(X^{1}, \ldots X^{m}\right), \quad 1 \leq \alpha \leq n .
$$

The increment vector $d x$ of point $q \in N$ in deformed body $N$ belongs to cotangent space $T_{q} N$, and the increment $d X$ of the corresponding point $X \in M$ in undeformed body $M$ belongs to tangent space $T_{p} M$. They are related by the differential map $F^{*}$.

Thus, the action of $F^{*}$ on the natural basis $\left\{d x^{\alpha}, 1 \leq \alpha \leq n\right\}$ is given by

$$
\begin{aligned}
F^{*}\left(\mathrm{~d} x^{\alpha}\right) & =d\left(x^{\alpha} \circ \mathrm{F}\right) \\
& =\sum_{1}^{m}\left(\frac{\partial F^{\alpha}}{\partial X^{i}}\right) \mathrm{d} X^{i} .
\end{aligned}
$$

The matrix representation of $F^{*}$ in the natural bases $d x$ and $d X$ is exactly the Jacobian matrix $\left(\partial F^{\alpha} \partial X^{i}\right)_{p}$. This relates the infinitesimal line segment $d X \in M$ to the corresponding segment $d x \in N$.

Map $F^{*}$ plays a role in the coordinate transformation from $T_{q} N$ to $T_{p} M$. Since no material point vanishes, there is an inverse relation for $F^{*}$ :

$$
\mathrm{d} x=F^{*-1}(\mathrm{~d} X),
$$

where $F^{*-1}$ is the deformation gradient.

A volume element $d V$ in undeformed body $M$ is related to the volume element $d v$ in deformed body $N$ through determinant $J$ of the deformation gradient $F$ by $d v=J d V$. 


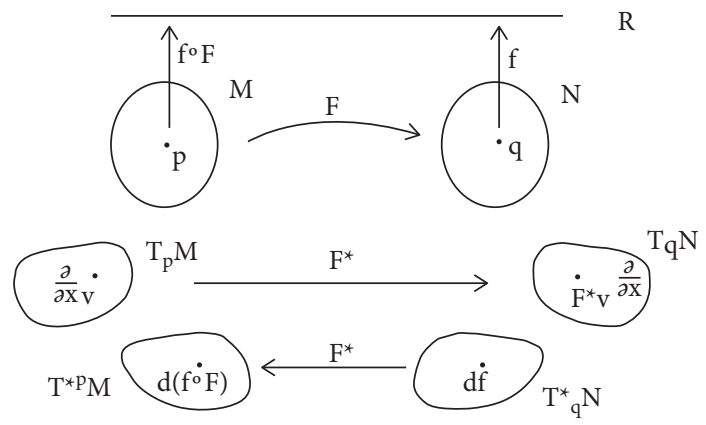

Figure 2: The tangent map and differential map. $T_{p} M$ and $T_{q} N$ are the tangent spaces. $T_{q}^{*}(\mathrm{~N})$ and $T_{p}^{*}(\mathrm{M})$ are the cotangent spaces.

$$
\begin{aligned}
J & =\operatorname{det} F \\
& =F^{*-1} F^{*-1} F^{*-1},
\end{aligned}
$$

where $J$ is referred to as the Jacobian.

The deformed body $N$ is a manifold with a topological structure and smooth structure. During deformation, some vectors act on it, such as body force and external force. They are vector bundles on $N$ (Figure 3 ).

$N$ is called the base space. $\pi: E \longrightarrow N$ is a smooth surjective map called a bundle projection, and $V=R^{s}$ is an s-dimensional vector space.

If there is no mass flux, the total mass of undeformed body $M$ is conserved in deformed body $N$ :

$$
\int_{M} \rho_{0} \mathrm{~d} v_{0}=\int_{N} \rho \mathrm{d} v
$$

where $\rho_{0}$ and $\rho$ are the mass densities before and after deformation, respectively. The time differential form using Reynold's transport theorem gives the following mass conservation law:

$$
\frac{\mathrm{d} \rho}{\mathrm{d} t}+\rho \nabla \cdot v=0
$$

where $v$ is a vector of the tangent bundle and $\nabla$ is the gradient operator. The divergence makes a linear map div: $T M \longrightarrow C^{\infty} M$.

Newton's second law states that in an inertial frame, the rate of linear momentum is equal to the applied force. Here, by applying the second law to a continuum region, the linear momentum of the deformed body $N$ is given by

$$
\frac{\mathrm{d}}{\mathrm{d} t} \int_{\Omega} \rho v \mathrm{~d} v=\int_{\partial \Omega} t \mathrm{~d} s+\int_{\Omega} \rho b \mathrm{~d} v
$$

where $t$ is an external force per unit area called the stress vector, which acts on the boundary $\partial \Omega$. It is a tensor of the tensor bundle $T_{0}{ }^{1}(N)$, and the body force per unit volume $b$ acting in volume $N$ is a tensor of tensor bundle TN.

Substituting (12) into (13) yields the following:

$$
\rho \frac{\mathrm{d} v}{\mathrm{~d} t}=\operatorname{div} \sigma^{T}+\rho b
$$

where $\sigma$ is called the Cauchy stress, which gives a transformation law that maps the unit outward $n$ to the traction $t$ acting on the surface by $t=\sigma^{T} n$. $\sigma$ is a tensor of tensor bundle $T_{0}{ }^{2}(N)$, and $n$ is a tensor of tensor bundle $T_{0}{ }^{1}(N)$.

Now, we need to measure the extent of deformation of an elemental length located at a material point. To do so, we should introduce a metric on the smooth manifold. If the metric is a smooth, nondegenerate positive symmetric second-order covariant tensor field, then the manifold is called a Riemannian manifold, and the metric is a Riemannian metric. We compare length $|d x|$ with its original length $|d X|$ by comparing the difference of both lengths as a squared measure:

$$
|\mathrm{d} x|^{2}-|\mathrm{d} X|^{2}=g_{i j} \mathrm{~d} x^{i} \mathrm{~d} x^{j}-G_{\alpha \beta} \mathrm{d} X^{\alpha} \mathrm{d} X^{\beta}
$$

where $g_{i j}$ is the metric tensor of manifold $N$, and $G_{\alpha \beta}$ is the metric tensor of manifold $M$.

Substituting the deformation gradient yields

$$
\begin{aligned}
|\mathrm{d} x|^{2}-|\mathrm{d} X|^{2} & =g_{i j} \mathrm{~d} x^{i} \mathrm{~d} x^{j}-G_{\alpha \beta} F_{i}^{* \alpha} F_{j}^{* \beta} \mathrm{d} x^{i} \mathrm{~d} x^{j} \\
& =\left(g_{i j}-G_{\alpha \beta} F_{i}^{* \alpha} F_{j}^{* \beta}\right) \mathrm{d} x^{i} \mathrm{~d} x^{j}
\end{aligned}
$$

Then, the deformation measure can be written as

$$
g_{i j}=2 \varepsilon_{i j}+G_{i j}
$$

This tensor $\varepsilon$ is referred to as a strain tensor.

Furthermore, $M$ and $N$ are two smooth manifolds, and $f$ : $M \longrightarrow N$ is a smooth map. If $f$ is the immersion and $h$ is a Riemannian metric, then

$$
g=f^{*} h
$$

where $g$ is the Riemannian metric of $M$. (17) indicates that the metric of manifold $M$ can be induced by the differential map and the metric of manifold of $N$.

The Riemann tensor $R$ of the initial configuration is a function:

$$
R_{i j k l}=\frac{1}{2}\left(G_{i k, j l}+G_{i l, j k}-G_{i l, j k}-G_{j k, i l}\right)+\widetilde{\Gamma}_{h . i k} \widetilde{\Gamma}_{j l}^{h}-\widetilde{\Gamma}_{h . i l} \widetilde{\Gamma}_{j k}^{h}
$$

The Riemann tensor $R$ of the current configuration is a function. 


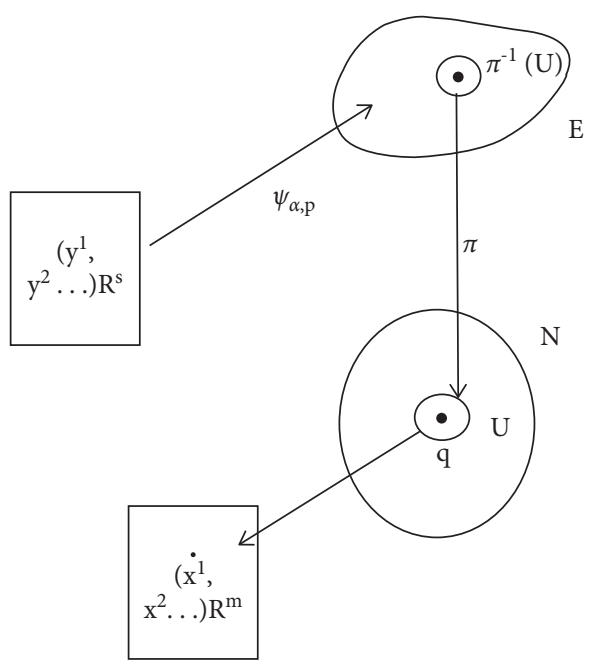

FIGURE 3: Vector bundles on the manifold. $E$ and $N$ are the smooth manifolds. $E$ is a vector bundle on $N$.

$$
R_{i j k l}=\frac{1}{2}\left(g_{i k, j l}+g_{i l, j k}-g_{i l, j k}-g_{j k, i l}\right)+\Gamma_{h . i k} \Gamma_{j l}^{h}-\Gamma_{h . i l} \Gamma_{j k}^{h}
$$

Substituting (18) and (16) into (19), equation (19) can be rewritten as

$$
R_{i j k l}=\left(\varepsilon_{i l, j k}+\varepsilon_{j k . i l}-\varepsilon_{i k, j l}-\varepsilon_{j l, i k}\right) .
$$

During excavation in a deep rock mass, a circular tunnel is a typical plane strain problem. Thus, the Riemann tensor $R$ can be obtained in a two-dimensional manifold as

$$
R_{1212}=2 \frac{\partial^{2} \epsilon_{12}}{\partial x^{1} \partial x^{2}}-\frac{\partial^{2} \varepsilon_{11}}{\partial x^{2} \partial x^{2}}-\frac{\partial^{2} \varepsilon_{22}}{\partial x^{1} \partial x^{1}} .
$$

The other component of $R_{i j k l}$ is equal to 0 . On the right of (21) is the compatible condition of classical mechanics. Due to the existence of closed internal stress, the deformation of rock masses is not compatible. Then, the tensor contraction of $R_{i j k l}$ is a second-order tensor $R_{i j}$, and the components are

$$
R_{i j}=\left|\begin{array}{cc}
\frac{R}{2} & 0 \\
0 & \frac{R}{2}
\end{array}\right|,
$$

where $R$ is the tensor contraction of $R_{i j}$, and $R=2 R_{1212}$.

\section{The Thermodynamics Model and Discussion}

Classical thermodynamics provide valuable information that can assist in the solving of practical problems in many fields of science and engineering. The physical state of the system can be described by variables such as internal energy, entropy energy, and free energy. They are decided by independent parameters; these parameters are the internal variables. The choice of the internal variables is not unique. It can be decided by the level of the model, the nature of the thermodynamic system, and the accuracy of the system state. The independent parameters consist of external variables (the strain tensor, deformation gradient, and temperature) that can be observed and internal variables that relate to the material properties of the system.

In geotechnical engineering, the strain tensor $\varepsilon$, temperature $T$, and Riemann tensor $R_{i j}$ can be chosen as external and internal variables. Now, we consider the deformation from the initial configuration to the final configuration, and this deformation is elastic. Thus, the Helmholtz free energy can be rewritten as

$$
\dot{\Psi}=\frac{\partial \Psi}{\partial T} \dot{T}+\frac{\partial \Psi}{\partial \epsilon_{i j}} \dot{\varepsilon}_{i j}+\frac{\partial \Psi}{\partial R_{i j}} \dot{R}_{i j}
$$

According to the second law of thermodynamics, the Clausius-Duhem inequality will be

$\rho \dot{T}\left(\frac{\partial \Psi}{\partial T}+s\right)+\rho \frac{\partial \Psi}{\partial \epsilon_{i j}} \dot{\varepsilon}_{i j}+\frac{\partial \Psi}{\partial R_{i j}} \dot{R}_{i j}-\sigma_{i j} \dot{\varepsilon}_{i j}+\frac{q}{T} \operatorname{div}, \quad T \leq 0$,

where $\rho$ is the density of the body, $s$ is the entropy, $q$ is the heat flux, and $\sigma_{i j}$ is the Cauchy stress tensor. The inequality above will be satisfied for any $T, Q, h$, and $\varepsilon$. Then, the following thermodynamic restrictions hold true:

$$
\begin{gathered}
\sigma_{i j}=\rho \frac{\partial \Psi}{\partial \epsilon_{i j}}, \\
\frac{\partial \Psi}{\partial R_{i j}} \dot{R}_{i j}+\frac{q}{T} \operatorname{div}, \quad T \leq 0, \\
s=-\frac{\partial \Psi}{\partial T} .
\end{gathered}
$$

Equation (25) indicates that the stress tensor is equal to the derived free energy, in accordance with classical elasticity theory.

Supposing that the rock material is isotropic at a constant temperature, we can extend the free energy to second order with

$$
\Psi\left(\varepsilon_{i j}, R_{i j}\right)=\frac{\lambda\left(\varepsilon_{k k} \varepsilon_{k k}\right)}{2}+\mu \varepsilon_{i j} \varepsilon_{i j}+\frac{\nu\left(R_{i j} R_{i j}\right)}{2}+\xi \varepsilon_{i j} R_{i j},
$$

where $\lambda$ and $\mu$ are Lame's constants, and $\nu$ and $\xi$ are the parameters related to the internal structure of the rock. Substituting equation (28) into (25) gives

$$
\sigma_{i j}=\lambda \varepsilon_{k k} \delta_{i j}+2 \mu \varepsilon_{i j}+\xi R_{i j}
$$

The underground rock experiences the effects of construction and heat, and the deformation will be incompatible and lead to a nonuniform stress field. A part of the stress will be lost by the tunnel excavation, but the residual will remain in the rock. In equation (29), for the initial undeformed body, the strain tensor is equal to zero. Then, the rock experiences an initial stress $\xi R_{i j}$, which is the self-balancing stress closed in the rock. This value is related to the metamorphic and tectonic 
movement of rock. From a physical point of view, the existence of nonzero self-equilibrated stress fields in a continuous medium is due to the presence of structural defects in the material.

The stress can be regarded as the sum of classical stress $\Sigma_{i j}$ and $t_{i j}$ :

$$
\sigma_{i j}=\Sigma_{i j}+t_{i j}
$$

According to the principles of thermodynamics with internal state variables, we define the force $Q$ as

$$
\begin{aligned}
Q & =-\frac{\partial \Psi}{\partial R_{i j}} \\
& =\nu R_{i j}+\xi \varepsilon_{i j},
\end{aligned}
$$

where $Q$ is a generalized force conjugated to $R$, which is an internal variable that relates to the internal stress of the rock mass and contributes to the dissipation of energy.

Recall that equation (29) can be written as

$$
\varepsilon_{i j}=\frac{1}{2 \mu}\left(\sigma_{i j}-\lambda \varepsilon_{k k} \delta_{i j}-\xi R_{i j}\right) .
$$

For the deformation from the initial configuration to the final configuration, the equilibrium and boundary conditions are

$$
\sigma_{i j, j}=0 \text {. }
$$

Substituting (32) and (33) into (21), we obtain

$$
R=\frac{2(\lambda+\mu)}{\mu(3 \lambda+2 \mu)} \Delta \sigma_{k k}-\frac{\lambda+2 \mu}{2 \mu(3 \lambda+2 \mu)} k \Delta R .
$$

Because the stress of the rock mass is self-balanced, $\Delta \sigma_{k k}=0$. Equation (34) can be rewritten as

$$
\Delta R+\omega R=0,
$$

where $\omega=2 \mu(3 \lambda+2 \mu) /(\lambda+2 \mu) \xi$. Equation (44) is Poisson's equation, and the solution is

$$
R=A J_{0}(\sqrt{\omega} \rho)+B Y_{0}(\sqrt{\omega} \rho)
$$

where $J_{0}$ and $Y_{0}$ are the Bessel function and Neumann function, respectively. $A$ and $B$ are the parameters related to the structural defects of the material.

Considering a circular tunnel in the plane strain state, the total stress can be written as

$$
\begin{gathered}
\sigma_{r}=p_{0}\left(1-\frac{r_{0}^{2}}{r^{2}}\right)+\xi A J_{0}(\sqrt{\omega} \rho)+\xi B Y_{0}(\sqrt{\omega} \rho), \\
\sigma_{\varphi}=p_{0}\left(1+\frac{r_{0}^{2}}{r^{2}}\right)+\xi A J_{0}(\sqrt{\omega} \rho)+\xi B Y_{0}(\sqrt{\omega} \rho) .
\end{gathered}
$$

The rock mass experiences gravitational stress and internal stress before excavation. The construction of underground engineering projects breaks the stress balance, and so, the stress will redistribute. In the plane strain state, the shear stress of the internal stress dissipates with the deformation, but normal stress still exists in the rock mass and shows a periodic change with radius. If this stress reaches a critical value, the rock will break.

\section{Analysis Based on Numerical Computations}

4.1. The Distribution of Internal Stress around a Deep Tunnel. The model for numerical computation [20-23] was from Tangkou mine coal, Shandong Province, China. During the excavation of the tunnel, the completed roadway was damaged successively, and the surrounding rock was fractured violently. The material parameters used in the simulations were from a depth of $1028 \mathrm{~m}$, including a uniaxial compressive strength of rock $\sigma_{c}=35 \mathrm{MPa}$, Young's modulus $E=30 \mathrm{GPa}$, and Poisson's ratio $v=0.25$. The cross-section of the tunnel is approximately a circle, and the radius is $r_{0}=3 \mathrm{~m}$. The stress of the surrounding rock is equivalent in vertical and horizontal orientations, and the stress $p_{0}=23.4 \mathrm{MPa}$.

Substituting these parameters into equations (37) and (38), we can obtain

$$
\begin{gathered}
\sigma_{r}=23.4\left(1-\frac{9}{r^{2}}\right)+\xi A J_{0}(3 \sqrt{\omega})+\xi B Y_{0}(3 \sqrt{\omega}), \\
\sigma_{\varphi}=23.4\left(1+\frac{9}{r^{2}}\right)+\xi A J_{0}(3 \sqrt{\omega})+\xi B Y_{0}(3 \sqrt{\omega}) .
\end{gathered}
$$

The parameter $\xi$ is related to the internal stress of the rock mass and can be chosen by the radius of the fracture zone. According to geological observations, the fracture zone radius $r_{c}=1.58 r_{0}$; thus, we have the following boundary conditions for the function $R$ :

$$
\left.R\right|_{r=r_{0}}=\left.0 \cdot \frac{d R}{d r}\right|_{r=r_{c}}=0 .
$$

Substituting (36) into (41) gives

$$
\begin{aligned}
A J_{0}(3 \sqrt{\omega})+B Y_{0}(3 \sqrt{\omega}) & =0, \\
\sqrt{\omega}\left[A J_{1}(4.74 \sqrt{\omega})+B Y_{1}(4.74 \sqrt{\omega})\right] & =0 .
\end{aligned}
$$

By solving equations (42) and (43), $\omega=7.18$ and $\xi=5.57 \mathrm{GPa}$ can be obtained.

Meanwhile, fracture appears when the stresses in the rock mass reach a certain critical value. From a physical viewpoint, this means that it is necessary to use a force criterion, whose fulfillment in a selected region corresponds to the fracture zone. In this study, the Mises criterion is applied.

$$
\left(\sigma_{1}-\sigma_{2}\right)^{2}+\left(\sigma_{2}-\sigma_{3}\right)^{2}+\left(\sigma_{3}-\sigma_{1}\right)^{2}=2 \sigma_{c}^{2} .
$$

Substituting (39) and (40) into (44), we obtain $A=5.05 \times 10^{-3}$ and $B=3.57 \times 10^{-3}$. Thus, the relation between the stress components and radius is shown in Figure 4. The distribution of hoop stress and radial stress shows a distinct wavy behavior. The range of fluctuation reflects the magnitude of internal stress and is related to in situ stress and rock behavior. 


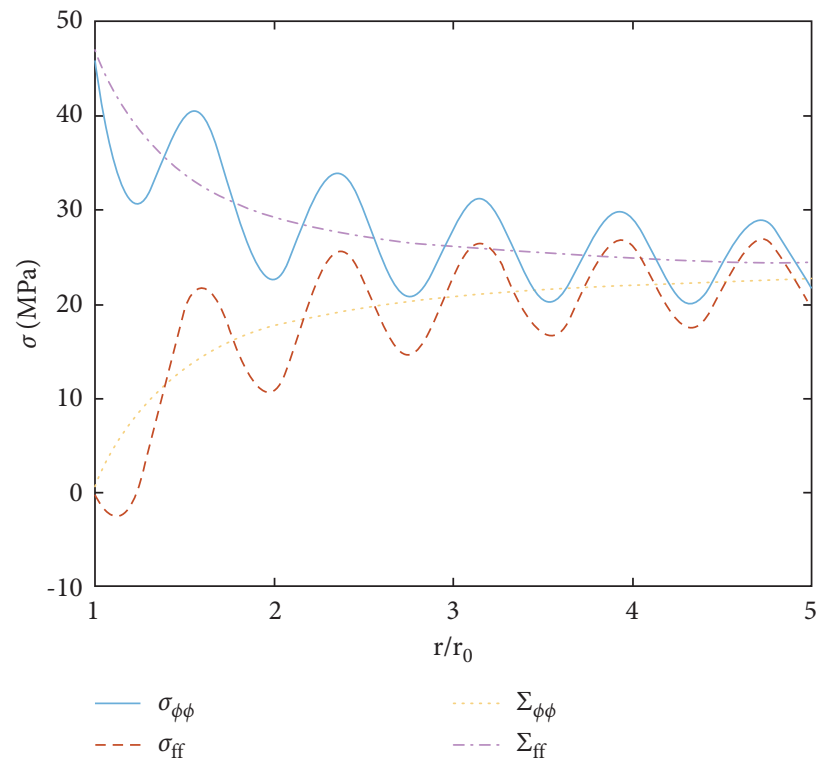

FIGURE 4: The distribution of stress with radius $(r)$.

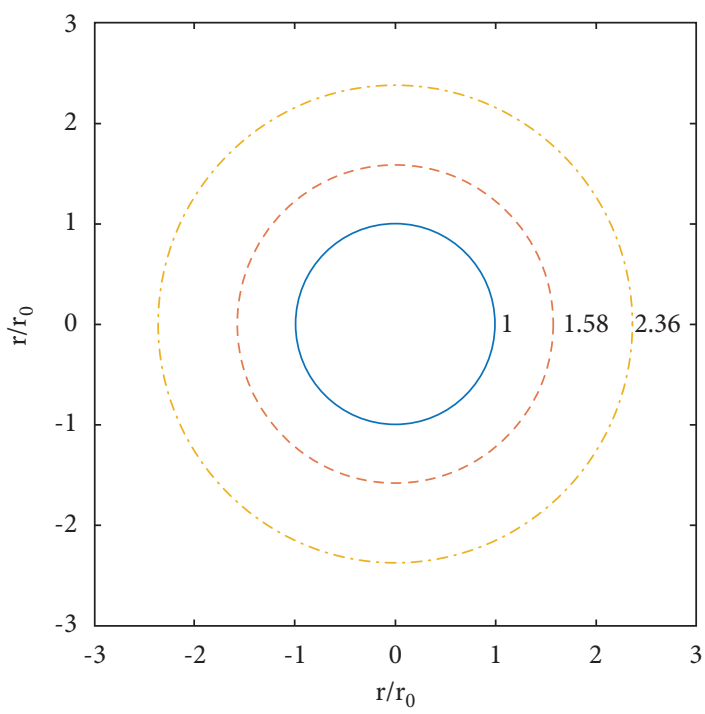

FIgURE 5: The site of crest zones from the computational model. Effects of rock parameters on the distribution of internal stress.

TABLE 1: Location of crest internal stress in the surrounding rock mass.

\begin{tabular}{|c|c|c|c|c|c|c|c|c|c|}
\hline \multirow[t]{2}{*}{ Location of crest stress $\left(\mathrm{r} / r_{0}\right)$} & \multicolumn{3}{|c|}{ Young's modulus E (GPa) } & \multicolumn{3}{|c|}{ Poisson's ratio $v$} & \multicolumn{3}{|c|}{$\begin{array}{c}\text { Non-Euclidean parameter } \xi \\
(\mathrm{GPa})\end{array}$} \\
\hline & 27 & 30 & 33 & 0.2 & 0.25 & 0.3 & 5.01 & 5.57 & 6.13 \\
\hline First & 1.69 & 1.58 & 1.47 & 1.63 & 1.58 & 1.46 & 1.43 & 1.58 & 1.67 \\
\hline Second & 2.37 & 2.36 & 2.02 & 2.29 & 2.36 & 2.06 & 2.01 & 2.36 & 2.34 \\
\hline
\end{tabular}

According to the prediction of the numerical computation, the second crest of the stress is located at $2.36 r_{0}$, and the observation value is $1.58 r_{0}$, as shown in Figure 5. The prediction value is $31 \%$ greater than the observation value because the fracture of the rock mass at $r=r_{c}$ is a region failure.
The fluctuation location and magnitude of the internal stress were influenced by the parameters of the rock mass, such as Young's modulus E, Poisson's ratio $v$, and nonEuclidean parameter $\xi$, which affected the fracture zone of the surrounding rock of the tunnel, and the location of the crest stress is given in Table 1. 


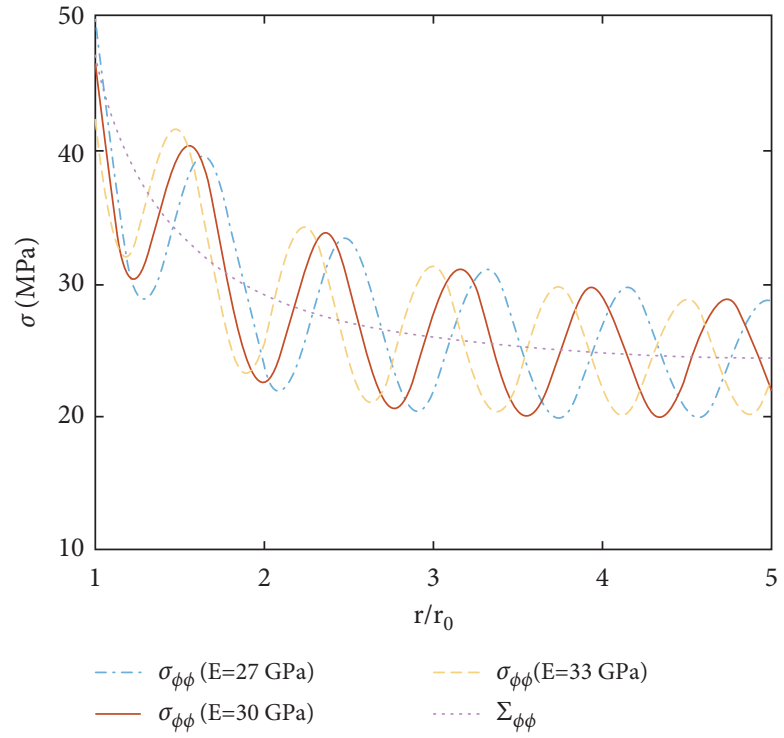

Figure 6: Effects of Young's modulus $(E)$ on the distribution of hoop normal stress in surrounding rock mass.

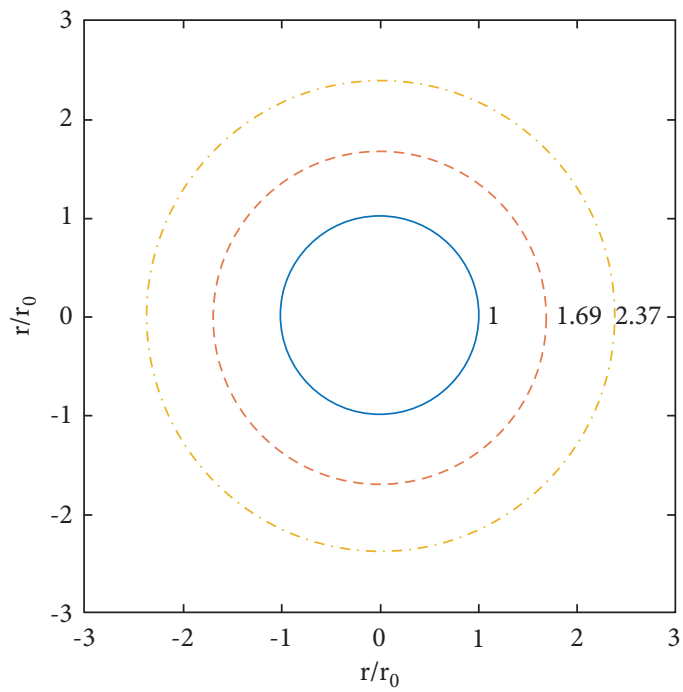

(a)

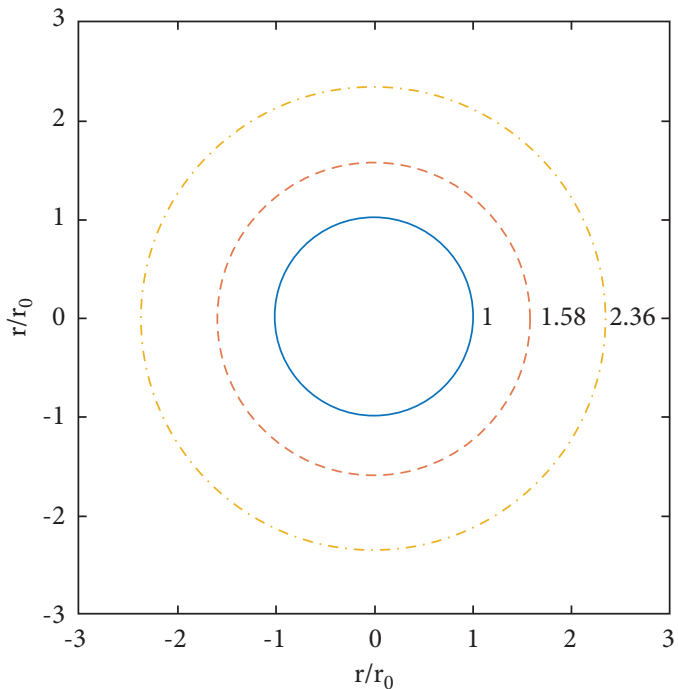

(b)

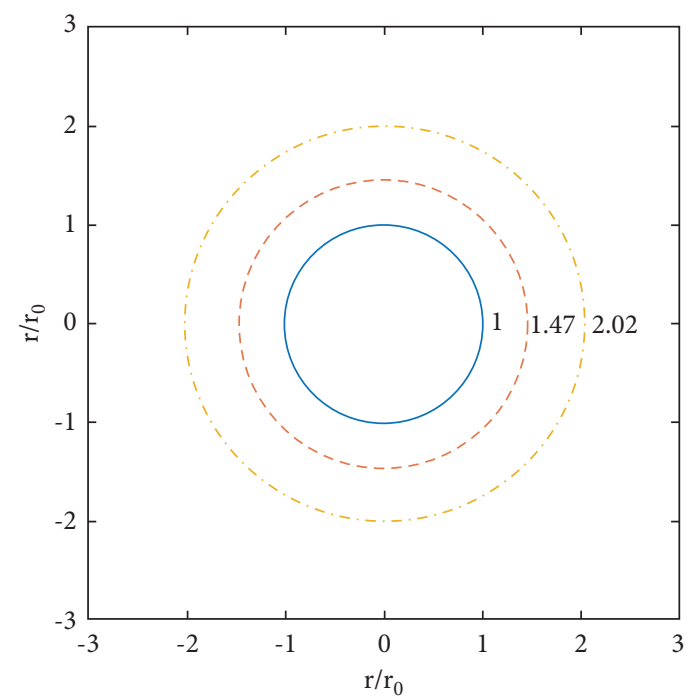

(c)

FIgURE 7: Location of crest internal stress in the surrounding rock mass with different Young's moduli. (a) $E=27 \mathrm{GPa}$. (b) $E=30 \mathrm{GPa}$. (c) $E=33 \mathrm{GPa}$. 


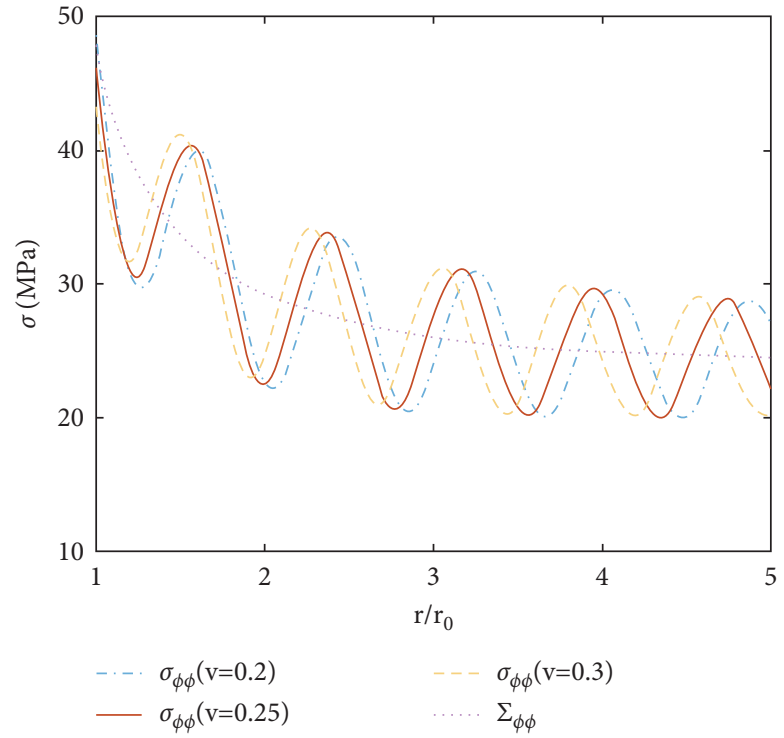

Figure 8: Effects of Poisson's ratio $(v)$ on the distribution of hoop normal stress in the surrounding rock mass.

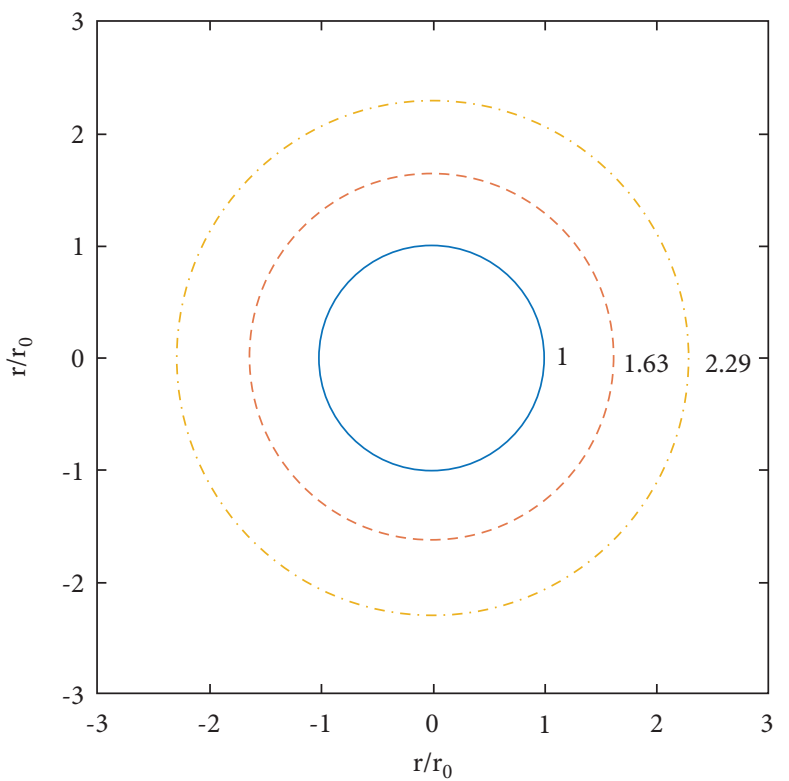

(a)

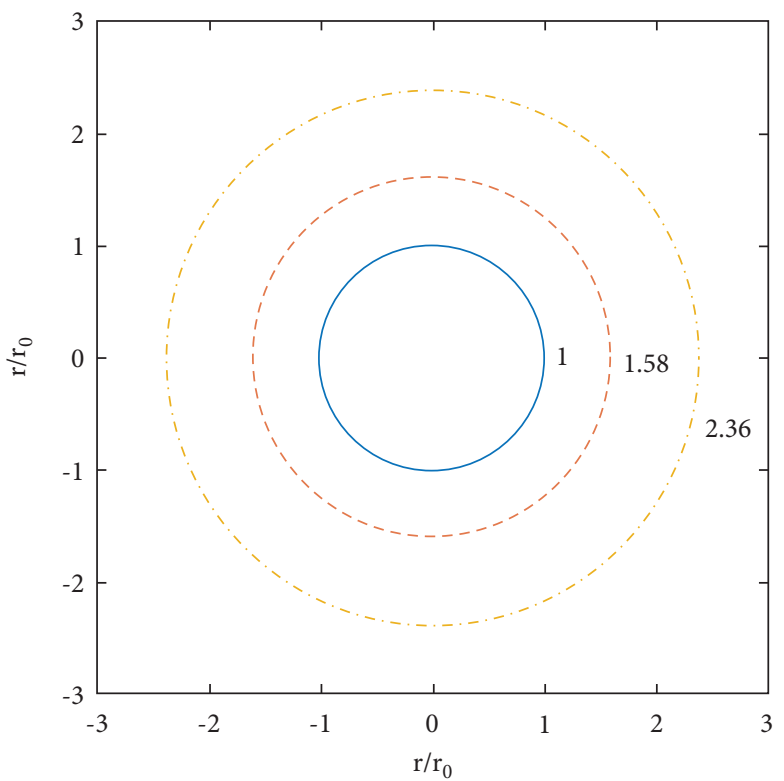

(b)

FIgure 9: Continued. 


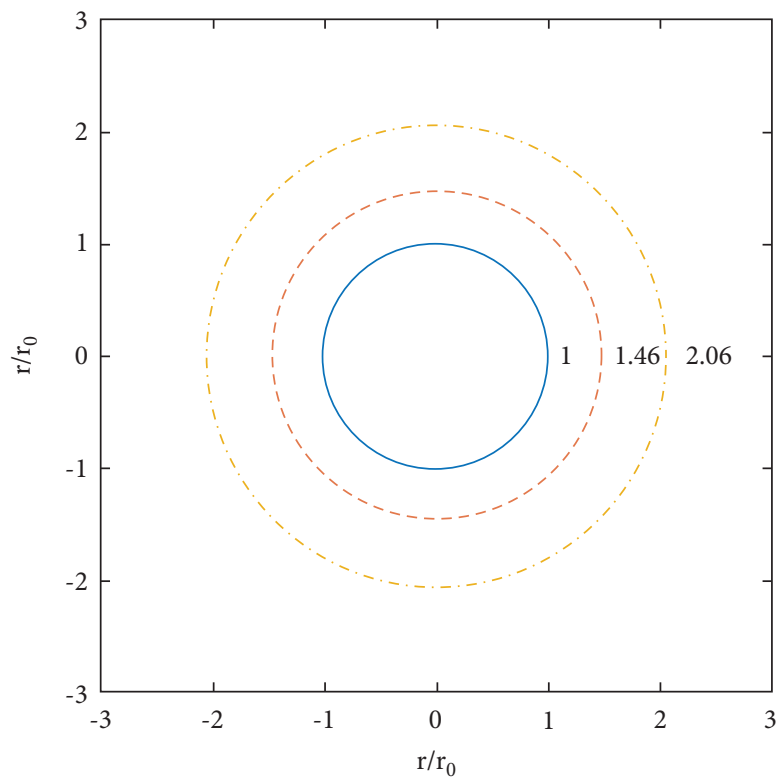

(c)

FIgURE 9: Location of crest internal stress in the surrounding rock mass with different Poisson's ratios. (a) $v=0.2$. (b) $v=0.25$. (c) $v=0.3$.

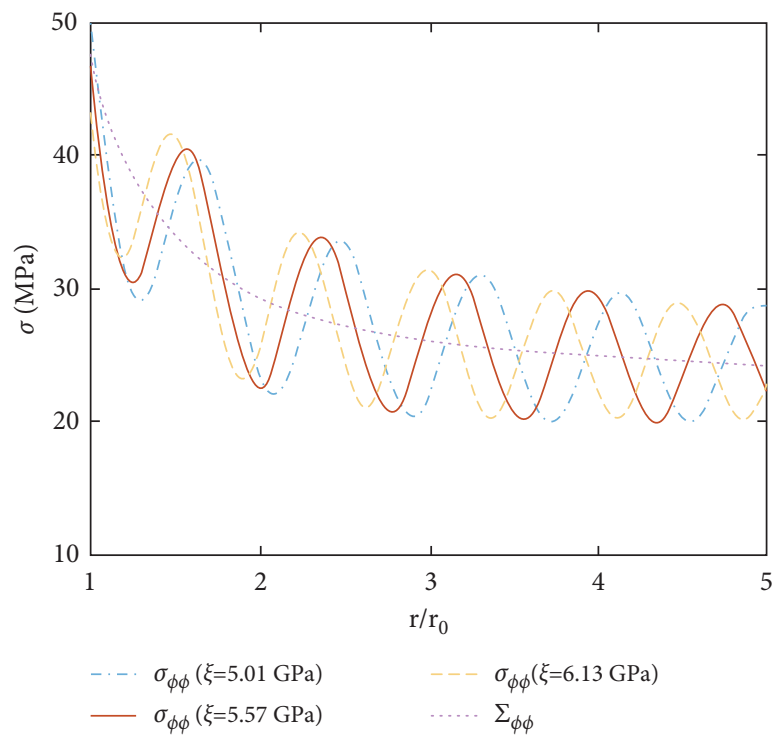

Figure 10: Effects of the non-Euclidean parameter $\xi$ on the distribution of hoop normal stress in surrounding rock mass.

Figures 6 and 7 show the effects of Young's modulus $E$ on the distribution of the internal stress crest in the surrounding rock mass around a deep circle tunnel. It can be observed that the radius of the internal stress crest decreases with an increase in Young's modulus $E$. These results indicate that the magnitude of internal stress and energy existing in the rock mass increases with Young's modulus. The larger Young's modulus is, the closer the fracture location is to the tunnel.
Figures 8 and 9 show the effects of Poisson's ratio $v$ on the distribution of the internal stress crest in the surrounding rock mass around a deep circle tunnel. The radius of fractured zones decreases with an increase in Poisson's ratio $v$.

Figures 10 and 11 show the effects of the non-Euclidean parameter $\xi$ on the distribution of the internal stress crest in the surrounding rock mass around a deep circle tunnel. It can be observed that the radius of 


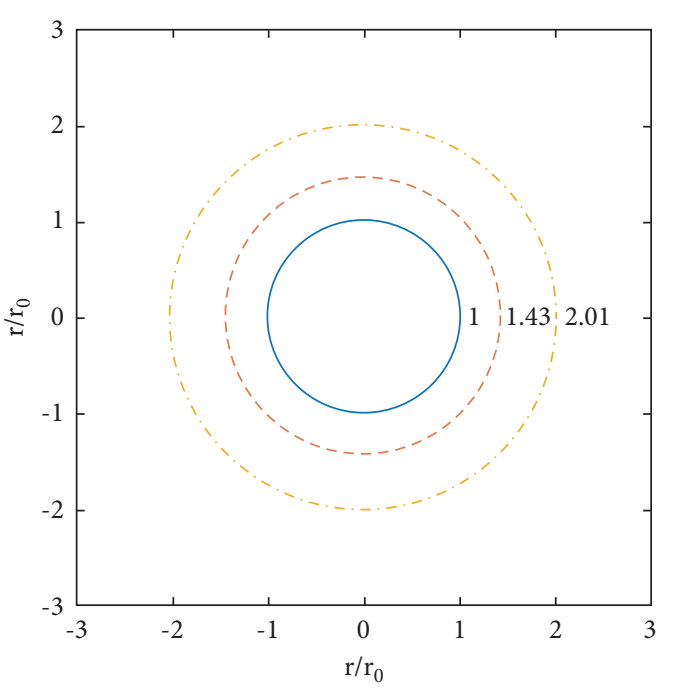

(a)

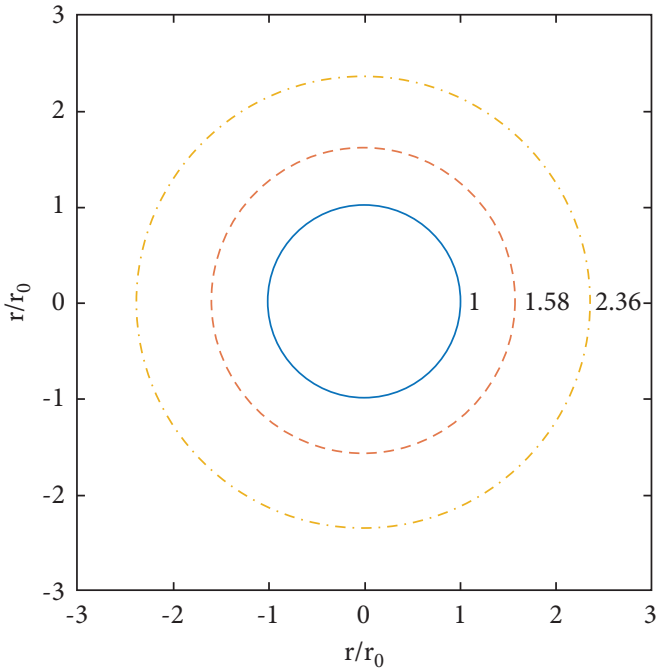

(b)

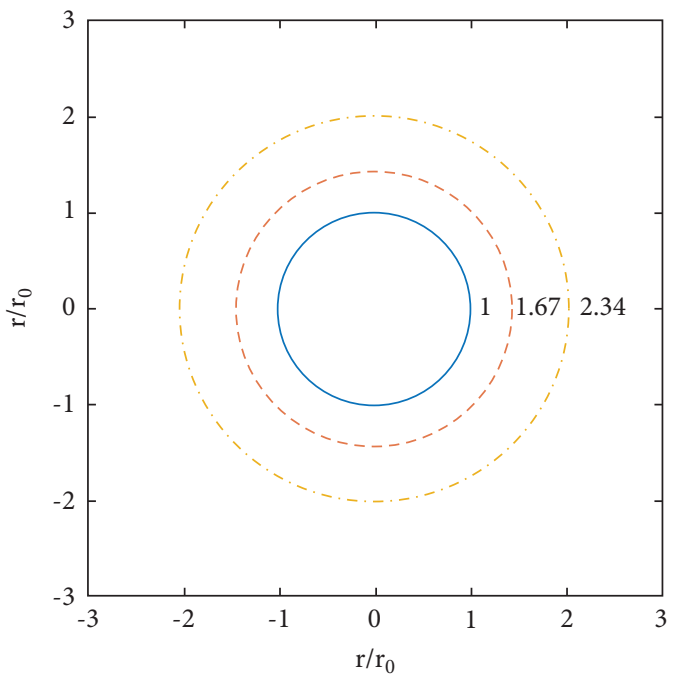

(c)

FIGURE 11: Location of the crest internal stress in surrounding rock mass with different non-Euclidean parameters. (a) $\xi=5.01 \mathrm{GPa}$. (b) $\xi=5.57 \mathrm{GPa}$. (c) $\xi=6.13 \mathrm{GPa}$.

fractured zones increases with an increase in the nonEuclidean parameter $\xi$.

\section{Conclusions}

(1) Because there is closed internal stress in the rock mass, the deformation of the rock mass is incompatible. The undeformed body is a manifold immersed in three-dimensional Euclidean space. The metric tensor of the space is the Riemannian metric $g_{i j}$ instead of the Euclidean metric $\delta_{i j}$. The deformation of the rock mass is a continuous map from the undeformed manifold $M$ to the deformed manifold $N$. The incompatible deformation of the rock mass is related to the Riemannian curvature tensor $R_{i j k l}$ of the manifold.

(2) The rock mass experiences gravitational stress and internal stress before excavation. The construction of underground engineering projects breaks the balance, and the stress will redistribute. In the plane strain state, the shear stress of the internal stress dissipates with the deformation, but normal stress still exists in the rock mass and shows a periodic change with radius. If this component reaches a critical value, the rock will break.

(3) For the rock mass with internal stress, with the increase of elastic modulus $E$, the peak value of hoop 
stress decreases, and the position of peak stress is closer to the tunnel. It shows that the harder the rock mass is, the more severe the damage near the tunnel surface is. With the increase of Poisson's ratio $v$, the hoop stress of rock mass decreases, which indicates that the larger Poisson's ratio $v$ is, the larger the deformation of rock mass under the same confining pressure, and more internal stress will be released. With an increase of non-Euclidean parameter $\xi$, the radius of the fractured zone decreases.

\section{Data Availability}

The data used to support the findings of this study are included within the article.

\section{Conflicts of Interest}

The authors declare that there are no conflicts of interest.

\section{Acknowledgments}

This work was financially supported by General Project of the National Natural Science Foundation of China (51174196).

\section{References}

[1] M. A. Guzev, "Non-classical solutions of a continuum model for rock descriptions," Journal of Rock Mechanics and Geotechnical Engineering, vol. 6, no. 3, pp. 180-185, 2014.

[2] M. Lazar, "Incompatible strain gradient elasticity of Mindlin type: screw and edge dislocations," Acta Mechanica, vol. 232, no. 9, pp. 3471-3494, 2021.

[3] W. Chunlai, He. Binbin, and H. Xiaolin, "Stress-Energy Mechanism for Rock Failure Evolution Based on Damage Mechanics," Hard Rock, vol. 3, pp. 1021-1037, 2020.

[4] Li. Zhipeng, W. Shunchuan, and C. Ziqiao, "Numerical Investigation of Dynamic Responses and Damage of Linings Subjected to Violent Gas Explosions inside Highway Tunnels," Shock and Vibration, vol. 2018, pp. 1-20, Article ID 2792043, 2018.

[5] E. V. Vtorushin and V. N. Dorovsky, "Application of nonstationary non-Euclidean model of inelastic deformations to rock cutting," Journal of Petroleum Science and Engineering, vol. 177, pp. 508-517, 2019.

[6] K. Kondo, "On the geometrical and physical foundations of the theory of yielding," in Proceedings of the 2nd National Congress on Applied Mechanics, pp. 41-47, Tokyo, Japan, June 1952.

[7] K.-H. Anthony, "Die theorie der Disklinationen," Archive for Rational Mechanics and Analysis, vol. 39, no. 1, pp. 43-88, 1970.

[8] E. Kröner, "Plastizität und Versetzungen," Mechanik der deformierbaren Medien, vol. 2, pp. 310-376, Akademische Arbeitsgemeinschaft Leipzig, Germany, 1964.

[9] R. De Wit, "A view of the relation between the continuum theory of lattice defects and non-Euclidean geometry in the linear approximation," International Journal of Engineering Science, vol. 19, no. 12, pp. 1475-1506, 1981.

[10] V. P. Myasnikov and M. A. Guzev, "Thermomechanical model of elastic-plastic materials with defect structures," Theoretical and Applied Fracture Mechanics, vol. 33, no. 3, pp. 165-171, 2000.
[11] M. A. Guzev and A. A. Paroshin, "Non-Euclidean model of the zonal disintegration of rocks around an underground working," Journal of Applied Mechanics and Technical Physics, vol. 42, no. 1, pp. 131-139, 2001.

[12] M. A. Guzev, "Structure of kinematic and force fields in the Riemannian continuum model," Journal of Applied Mechanics and Technical Physics, vol. 52, no. 5, pp. 709-716, 2011.

[13] V. V. Makarov, M. A. Guzev, V. N. Odintsev, and L. S. Ksendzenko, "Periodical zonal character of damage near the openings in highly-stressed rock mass conditions," Journal of Rock Mechanics and Geotechnical Engineering, vol. 8, no. 2, pp. 164-169, 2016.

[14] M. A. Guzev, V. N. Odintsev, and V. V. Makarov, "Principals of geomechanics of highly stressed rock and rock massifs," Tunnelling and Underground Space Technology, vol. 81, pp. 506-511, 2018.

[15] M. A. Guzev and V. V. Makarov, "Physical mesomechanics approach to modeling the earth's subsurface," Physical Mesomechanics, vol. 24, no. 4, pp. 357-362, 2021.

[16] Q. Qian and X. Zhou, "Non-Euclidean continuum model of the zonal disintegration of surrounding rocks around a deep circular tunnel in a non-hydrostatic pressure state," Journal of Mining Science, vol. 47, no. 1, pp. 37-46, 2011.

[17] X. P. Zhou, H. F. Song, and Q. H. Qian, "Zonal disintegration of deep crack-weakened rock masses: a non-Euclidean model," Theoretical and Applied Fracture Mechanics, vol. 55, no. 3, pp. 227-236, 2011.

[18] X. Zhou, Q. Hou, Q. Qian, and Y. Zhang, "The zonal disintegration mechanism of surrounding rock around deep spherical tunnels under hydrostatic pressure condition: a non-Euclidean continuum damage model," Acta Mechanica Solida Sinica, vol. 26, no. 4, pp. 373-387, 2013.

[19] Y. D. Shou, X. P. Zhou, and Q. H. Qian, "A critical condition of the zonal disintegration in deep rock masses: strain energy density approach," Theoretical and Applied Fracture Mechanics, vol. 97, pp. 322-332, 2018.

[20] S.-S. Chern, W.-h. Chen, and K. S. Lam, Lectures on Differential Geometry, World Scientific Publishing Company, Singapore, 1999.

[21] R. Pakzad, S. Wang, and S. W. Sloan, "Three-dimensional finite element simulation of fracture propagation in rock specimens with pre-existing fissure(s) under compression and their strength analysis," International Journal for Numerical and Analytical Methods in Geomechanics, vol. 44, no. 10, pp. 1472-1494, 2020.

[22] C. D Norton, V. K Decyk, B. K Szymanski, and H Gardner, "The transition and adoption to modern programming concepts for scientific computing in Fortran," Scientific Programming, vol. 15, Article ID 146752, 18 pages, 2007.

[23] Y. A Fageehi and A. M Alshoaibi, "Nonplanar crack growth simulation of multiple cracks using finite element method," Advances in Materials Science and Engineering, vol. 2020, Article ID 8379695, 12 pages, 2020. 